\title{
Water in Olive Oil Surfactantless Microemulsions as Medium for CdS Nanoparticles Synthesis
}

\author{
Mohamed E. EL-Hefnawy \\ Department of Chemistry, Faculty of Science \\ Tanta University, Tanta 31527, Egypt \\ Tel: 20-1-9270-9889 E-mail: mhefnawy46@yahoo.com
}

Received: February 20, 2012

Accepted: March 12, $2012 \quad$ Published: April 1, 2012

doi:10.5539/mas.v6n4p101

URL: http://dx.doi.org/10.5539/mas.v6n4p101

\begin{abstract}
A simple chemical route using ternary 1-butanol/olive/water microemulsion systems without surfactant has been developed to synthesize spherical CdS nanoparticles with an average diameter of ca. $45 \mathrm{~nm}$. The CdS nanoparticles were investigated by transmission electron microscopy (TEM), UV-VIS optical absorption spectrum and Fourier transform infrared (FTIR) spectroscopy measurements. By comparision with previous work, the present work confirmed the effective role of the surfactant in formation nanoparticles.
\end{abstract}

Keywords: Cadmium sulfide, Nanoparticle, Transmittance electron microscope, Infrared spectroscopy

\section{Introduction}

At the moment nanostructural materials have become attractive because of their unique characteristics that can hardly be obtained from conventional bulk materials (Alivisatos, 1996) owing to their quantum size and surface effects. In particular, much attention has been paid to synthesis of semiconductor materials due to their excellent prospective in optical, electronic and magnetic functionality, and so on (El-Sayed, 2004).

To date there are many methodologies available for synthesizing CdS nanocrystals, such as soft chemical reaction, solid-state reaction, sol-gel process, sonochemical preparation (Mane, et al., 2000; Henshaw, et al., 1996), microwave heating (Wada, et al., 2001), photoetching (Torimoto, et al., 2001). However, water-in-oil (w/o) microemulsions or reverse micelles technique is one of the most recognized methods due to its several advantages, for instance, soft chemistry, demanding no extreme pressure or temperature control, easy to handle, and requiring no special or expensive equipment. Synthesis of nanoparticles in water-in-oil microemulsions, can yield particles nearly monodisperse in size (Ethayaraja, et al., 2007; Caponetti, et al., 2006; Tan, et al., 2011). In this investigation, we have developed a method to produce CdS nanoparticles of small sizes using new microemulsions systems without surfactant. These ternary mixtures consisting of an inexpensive non-toxic oil (Olive oil), a short chain-alcohol and water represent thermodynamically stable dispersion of nanometer-sized water drops in a continuous oil medium. There are two motivations behind this work. One due to the literature concerning the preparation of CdS nanoparticles by use of water-in-oil microemulsion without surfactant is quite scarce (Ghows, et al., 2011). The other for continuation my previous work for study the roles of surfactant and cosurfactant in the formation of CdS nanoparticles (EL-Hefnawy, 2012). Thereby, in this paper, CdS nanoparticles were prepared in ternary 1-butanol/olive/water microemulsion. CdS nanoparticles are extensively characterized using TEM, UV-VIS spectrophotometer, and FTIR spectroscopy. The structural aspects of this surfactantless microemulsion were discussed by the camparison with my previous work.

\section{Experimental}

\subsection{Materials}

Virgin olive oil was obtained from the local market. 1-buanol (99\% pure) was purchased from Sigma. Cadmium chloride and sodium sulfide were purchased from Merck. These reagents were used as received. Deionized Millipore Milli-Q water was used in all the experiments.

\subsection{Construction of Phase Diagram}

In order to find the concentration range of all components [olive oil/1-butanol/water] in which they form surfactantless microemulsions, phase diagram was constructed using the water titration method. Olive oil and 
1-butanol were mixed at the weight ratios of 1:9, 2:8, 3:7, 4:6, 5:5, 6:4, 7:3, 8:2, and 9:1. These mixtures were diluted dropwise with water, under moderate agitation. The samples were classified as microemulsions when they appeared as clear liquids. Phase diagram is presented in Figure 1.

\subsection{Synthesis of CdS Nanoparticles}

To prepare the microemulsion, the oil phase and 1-butanol were mixed by magnetically stirring until the mixture became transparent. Divide the mixture into two parts, and the two different microemulsions were prepared as follows: the $\mathrm{CdCl}_{2}$ aqueous $(0.012 \mathrm{M})$ was added into one of the parts then the reverse microemulsion of $\mathrm{CdCl}_{2}$ was obtained; the $\mathrm{Na}_{2} \mathrm{~S} .9 \mathrm{H}_{2} \mathrm{O}$ aqueous $(0.012 \mathrm{M})$ was added into the other part of mixture until the mixture became transparent, then the reverse microemulsion of $\mathrm{Na}_{2} \mathrm{~S}$ was prepared. All experimental processes were under magnetic stirring. The two reverse microemulsions were mixed and magnetically stirred at room temperature of $27^{\circ} \mathrm{C}$ for about $15 \mathrm{~min}$.

\subsection{Characterization}

TEM was used to examine the morphology of the CdS nanoparticles on a JEOL transmission electron microscope (model: JEM- 1230, Japan operating at $120 \mathrm{kV}$ attached to a CCD camera). The particle size values were automatically registered from a programm of Gatan camera digital micrograph software. The UV-Vis spectra were recorded in a range form 200 to $1000 \mathrm{~nm}$ using Shimadzu UV-1601PC UV-visible spectrophotometer. Microemulsion solution without any salt was used as a reference for UV-visible absorption measurements. When equal volumes of microemulsions containing $\mathrm{CdCl}_{2}$ and $\mathrm{Na}_{2} \mathrm{~S}$ were mixed in a cuvette, a deep yellow color instantaneously appeared, confirming the formation of CdS nanoparticles. Fourier transform infrared (FT-IR) measurements were carried out using Perkin Elmer Spectrum1 FT-IR with a resolution of 4 $\mathrm{cm}^{-1}$.

\section{Results and Discussion}

The TEM images of CdS nanoparticles prepared at reverse microemulsion system are shown in Figure 2. It can be seen that nanoparticles have spherical morphology with an average diameter of ca. $45 \mathrm{~nm}$. We can find from this figure that the morphology of the particles is almost homogeneous. In the previous work (EL-Hefnawy, $2012)$, it was observed that microemulsion with the ratio of water to surfactant $\left(\omega_{0}\right)=5$ was suitable to get the finest particle size $16 \mathrm{~nm}$. It was significant to increase $\omega_{\mathrm{o}}$ from 5 to 15 as the particles increases gradually in this response. Thereby, the surfactant with $\omega_{0}=10$ and 15 is unable to prevent the agglomeration of CdS particles. This observation will be more confirmed in the present work. In the present work, CdS nanoparticles were prepared in ternary 1-butanol/olive/water microemulsion without surfactant with aqueous phase $\%=3.9$ that is identical to the ratio of aqueous phase in microemulsion with $\omega_{0}=5$ in the previous work. Compared with the results obtained from this previous work, the present results demonstrate that this method can fabricate CdS nanoparticles with large dimensions (average diameter ca. $45 \mathrm{~nm}$ ). This result confirms that the surfactant (SDS) plays a critical role in the formation of CdS nanoparticles with small dimensions. SDS and 1-butanol adheres to the surface of the nanoparticles served as a protective layer to prevent them from aggregating to larger particles (Sun, et al., 2002, Weller, et al., 2002; Mei, et al., 2006; Huang, et al., 2004; Vidal, et al., 2006). This conclusion is further support to previous results (EL-Hefnawy, 2012), and interprets why the synthesized CdS nanoparticles in surfactantless microemulsions were formed with large dimensions. The absence of SDS in this work left 1-butanol to adhere alone with nanoparticles. Thereby, 1-butanol was unable alone to prevent the agglomeration of CdS particles.

Figure 3 shows the UV-VIS optical absorption spectrum of CdS nanoparticles synthesized in surfactantless microemulsion. We can estimate that an UV-VIS optical absorption excitonic peak of the nanoparticles is at ca. $330 \mathrm{~nm}$, and shows a blue shift from that of bulk CdS $(530 \mathrm{~nm})$ crystals. This result is in agreement with the value of the reported literatures (EL-Hefnawy, 2012; Henglein, et al., 1989).

FTIR measurement has been made in the wave number range $400 \mathrm{~cm}^{-1}$ to $4000 \mathrm{~cm}^{-1}$. The FTIR spectra is shown in Figure 4. CdS particles showed two stretching bands, asymmetric and symmetric, around $2920.2 \mathrm{~cm}^{-1}$ and $2852.6 \mathrm{~cm}^{-1}$ are associated with $\mathrm{C}-\mathrm{H}$ stretching. There are weak to medium absorption bands at $630 \mathrm{~cm}^{-1}$ and 725 $\mathrm{cm}^{-1}$ possibly due to Cd-S stretching. Hence the existences of above mentioned bands identify the presence of CdS.

\section{References}

Alivisatos, A. P. (1996). Perspectives on the physical chemistry of semiconductor nanocrystals. J. Phys. Chem., 100(31), 13226-13239. http://dx.doi.org/10.1021/jp9535506

Caponetti, E., Martino, D. C., Leone, M., Pedone, L., Saladino, M. L., \& Vetri, V. (2006). Microwave-assisted 
synthesis of anhydrous CdS nanoparticles in Sci. a water-oil microemulsion. J Colloid Interface, 304(2), 413-418. http://dx.doi.org/10.1016/j.jcis.2006.09.026

EL-Hefnawy, M. E. (2012). Water in olive oil microemulsions as medium for CdS nanoparticles synthesis. $J$. Chil. Chem. Soc., submitted.

El-Sayed, M. A. (2004). Small Is Different: Shape-, Size-, and Composition-Dependent Properties of Some Colloidal Semiconductor Nanocrystals. Acc. Chem. Res., 37(5), 326-333. http://dx.doi.org/10.1021/ar020204f

Ethayaraja, M., Dutta, K., Muthukumaran, D., \& Bandyopadhyaya, R. (2007). Nanoparticle Formation in Water-in-Oil Microemulsions: Experiments, Mechanism, and Monte Carlo Simulation. Langmuir, 23(6), 3418-3423. http://dx.doi.org/10.1021/la062896c

Ghows, N., \& Entezari, M. H. (2011). A novel method for the synthesis of CdS nanoparticles without surfactant. Ultrasonics Sonochemistry, 18(1), 269-275. http://dx.doi.org/10.1016/j.ultsonch.2010.06.008

Henglein, A. (1989). Small-particle research: physicochemical properties of extremely small colloidal metal and semiconductor particles. Chem. Rev., 89(8), 1861-1873. http://dx.doi.org/10.1021/cr00098a010

Henshaw, G., Oarkin, I. P., \& Shaw, G. (1996). Convenient, low-energy synthesis of metal sulfides and selenides; $\mathrm{PbE}, \quad \mathrm{Ag} 2 \mathrm{E}, \mathrm{ZnE}, \quad \mathrm{CdE}(\mathrm{E}=\mathrm{S}, \mathrm{Se})$. Chem. Communs, 27, 1095-1096. http://pubs.rsc.org/en/content/articlelanding/1996/cc/cc9960001095

Huang, Y., Ma, T., Yang, J. L., Zhang, L. M., He, J. T., \& Li, H. F. (2004). Preparation of spherical ultrafine zirconia powder in microemulsion system and its dispersibility. Ceramics International, 30, 675-681. http://dx.doi.org/10.1016/j.ceramint.2003.06.001

Mane, R. S., \& Lokhande, C. D. (2000). Chemical deposition method for metal chalcogenide thin films. Mater. Chem. Phys., 65, 1-31. http://dx.doi.org/10.1016/S0254-0584(00)00217-0

Mei, Y., Han, Y. B., Li, Y., Wang, W., \& Nie, Z. R. (2006). Measurement of microemulsion zone and preparation of monodispersed cerium oxide nanoparticles by W/O microemulsion method. Materials Letters, 60, 3068-3072. http://dx.doi.org/10.1016/j.matlet.2006.02.044

Sun, L. D., Zhang, Y. X., Zhang, J., Yan, C. H., Liao, C. S., \& Lu, Y. Q. (2002). Fabrication of size controllable YVO4 nanoparticles via microemulsion-mediated synthetic process. Solid State Communications, 124, 35-38. http://dx.doi.org/10.1016/S0038-1098(02)00449-0

Tan, T. T. Y., Liu, S., Zhang, Y., Han, M. Y., \& Selvan, S. T. (2011). Microemulsion Preparative Methods (Overview). Comprehensive Nanoscience and Technology, 5, 399-441. http://www.sciencedirect.com/science/article/pii/B9780123743961000453

Torimoto, T., Kontani, H., Shibutani, Y., Kuwabata, S., Sakata, T., Mori, H., \& Yoneyama, H. (2001). Characterization of Ultrasmall CdS Nanoparticles Prepared by the Size-Selective Photoetching Technique. $J$. Phys. Chem., 105, 6838-6845. http://dx.doi.org/10.1021/jp0109271

Vidal, V. J., Rivas J., \& Lopea-ouintela, M. A. (2006). Synthesis of monodisperse maghemite nanoparticles by the microemulsion method. Colloids and Surfaces A: Physicochemical and Engineering Aspects, 288, 44-51. http://dx.doi.org/10.1016/j.colsurfa.2006.04.027

Wada, Y., Kuramoto, H., Anand, J., Tikamura, T., Sakata, T., Mori H., \&Yanagida, S. (2001). Microwave assisted size control of CdS nanocrystallites. J. Mater. Chem., 11, 1936-1940. http://dx.doi.org/10.1039/b101358k

Weller, H., Schmidt, H. M., Koch, U., Fojtik, A., Baral, S., Henglein, A., Kunath, W., Weiss, K., \& Dieman, E. (1986). Photochemistry of colloidal semiconductors. Onset of light absorption as a function of size of extremely small CdS particles. Chem. Phys. Lett., 124(6), 557-560. http://dx.doi.org/10.1016/0009-2614(86)85075-8 


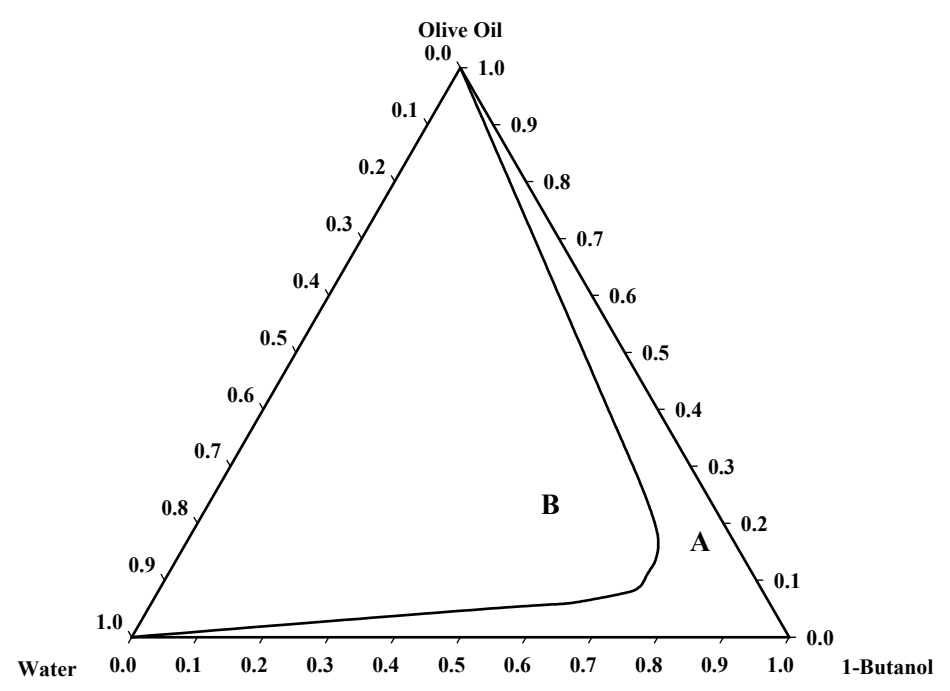

Figure 1. Phase diagram of water/ oil/ 1-butanol mixtures at $27 \mathrm{oC}$. The area A indicates the microemulsions domain.

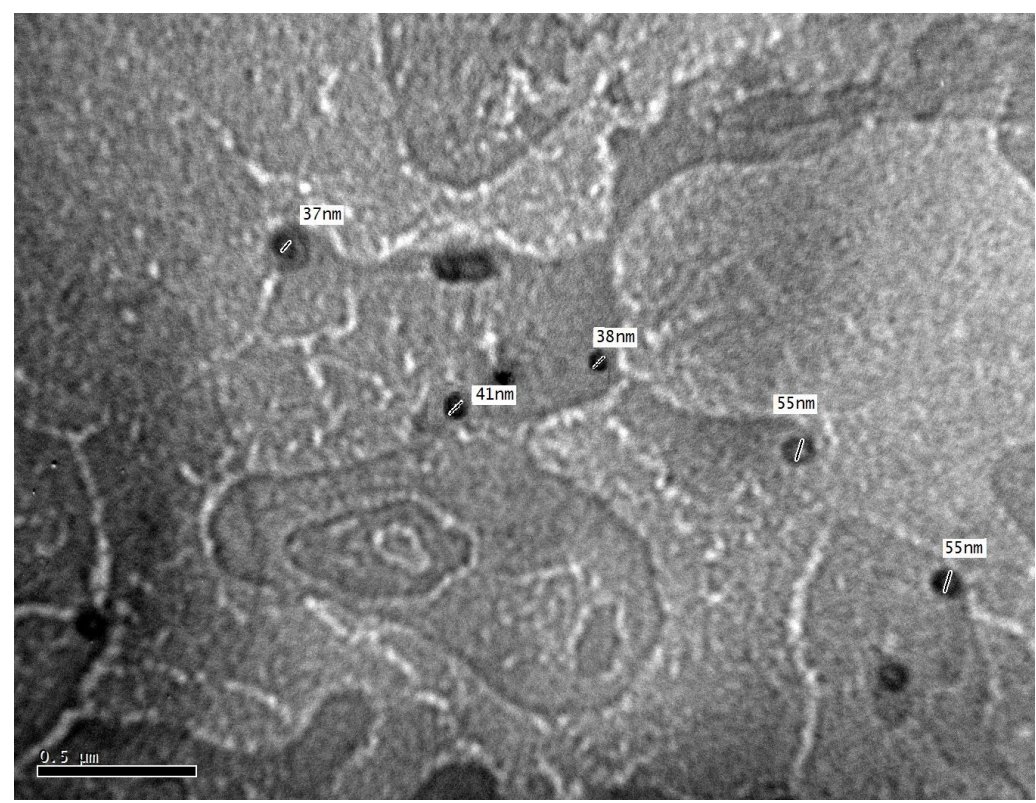

Figure 2. TEM images CdS nanoparticles in microemulsion at aqueous phase $\%=3.9$ 


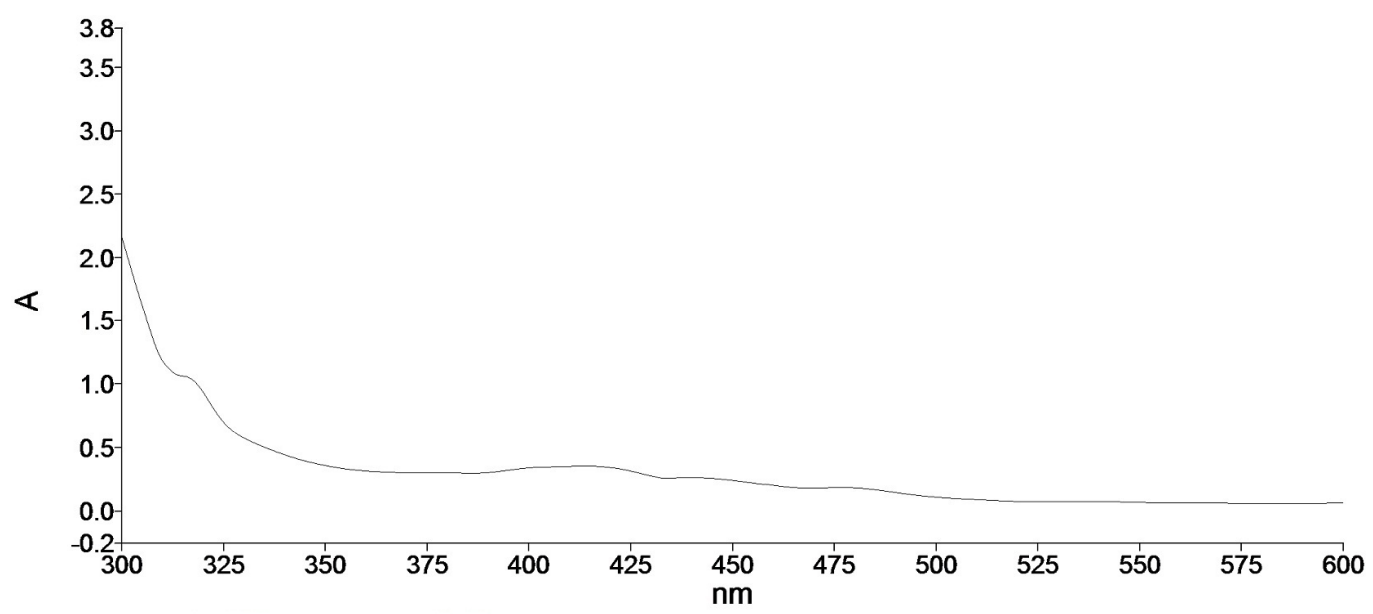

Figure 3. UV- VIS absorption spectrum of the CdS nanoparticles in 1-butanol/Olive oil/water microemulsion with aqueous phase $\%=3.9$

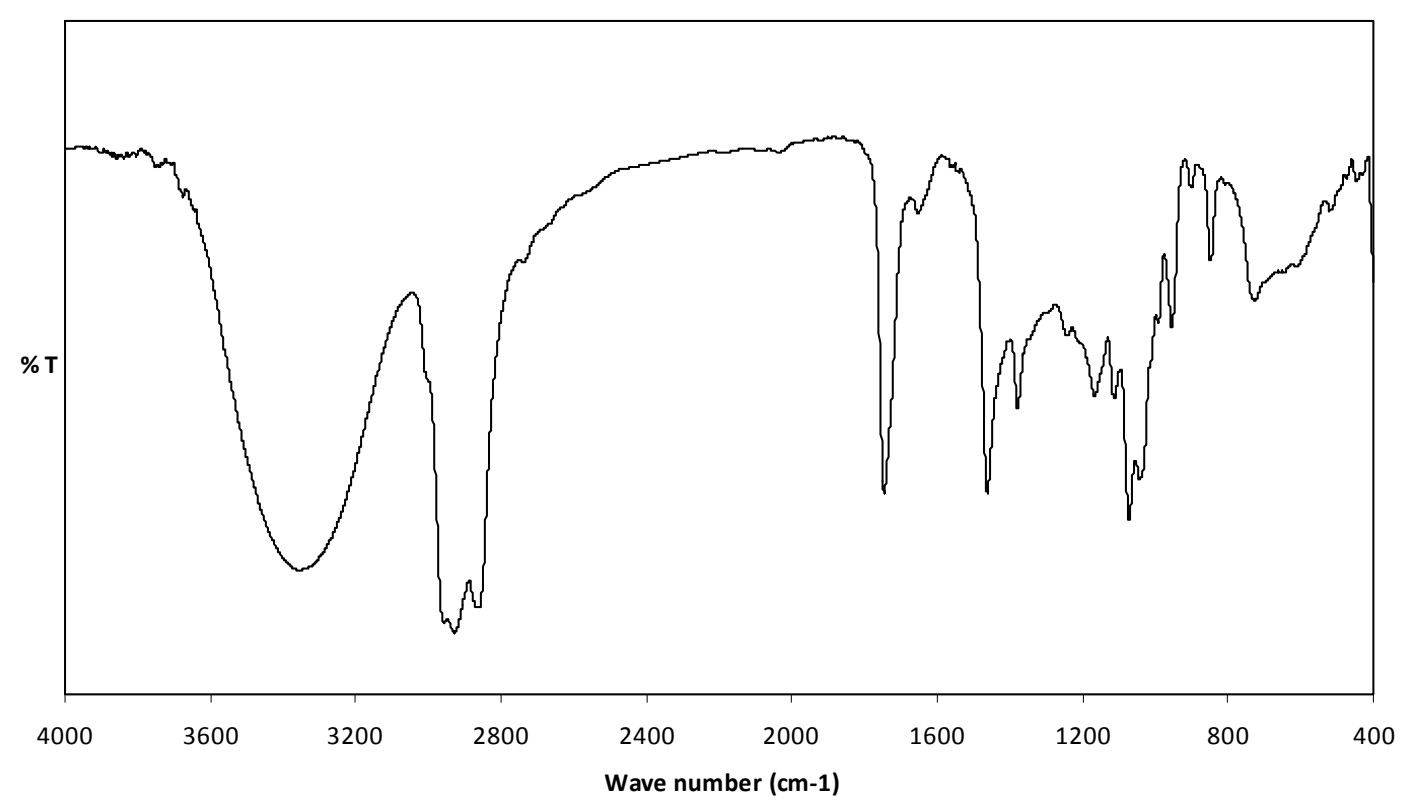

Figure 4. IR spectra of the CdS nanoparticles in 1-butanol/Olive oil/water microemulsion with aqueous phase\% $=3.9$ 